\title{
Local Shwartzman Reaction in the Rat Induced by Endotoxin and Ischemia: Potential Model for Skin Necrosis in Meningococcemia
}

\author{
ANTHONY J. PENINGTON, RANDALL O. CRAFT, GERALDINE MITCHELL, AND WAYNE A. MORRISON \\ The Bernard O'Brien Institute of Microsurgery [A.J.P., R.O.C., G.M., W.A.M.], St. Vincent's Hospital, Fitzroy, Victoria 3065, Australia; \\ Department of Surgery [A.J.P., R.O.C., W.A.M.], University of Melbourne, Victoria 3010, Australia
}

\begin{abstract}
The local Shwartzman reaction (LSR) is an inflammatory response in the skin that is considered a model for skin necrosis associated with meningococcemia. We tested the hypothesis that ischemia can act as the provocative agent to produce this response. In eight rats, bilateral inferior epigastric flaps were outlined. Within each flap, three injection sites were marked. Site 1 had $0.1 \mathrm{~mL}$ of endotoxin injected $24 \mathrm{~h}$ before surgery. The other two sites had either endotoxin or saline injected immediately before surgery. Both flaps were raised on their pedicle and one side rendered ischemic for $6 \mathrm{~h}$ and then reperfused. Animals were killed either 30 min or $48 \mathrm{~h}$ later and the tissue from each site examined. After $48 \mathrm{~h}$ of reperfusion, necrosis was grossly visible at the site of 24-h preischemia injection of endotoxin in three of four rats. No abnormalities were present at the other injection sites. Microscopically, all 24-h-delayed injection sites showed hemorrhage into all layers of the skin after both $30 \mathrm{~min}$ and $48 \mathrm{~h}$ of reperfusion. No hemorrhage was present at the other sites. These findings may serve as a potential model for the skin necrosis seen in meningococcemia. (Pediatr Res 60: 158-160, 2006)
\end{abstract}

$\mathrm{T}$ he LSR is an inflammatory response in the skin that is considered a model for the skin necrosis associated with meningococcemia. It is classically induced in the skin of rabbits by an intradermal injection of bacterial lipopolysaccharide followed by an intravenous provocative dose $18-36 \mathrm{~h}$ later. The resulting LSR is initially seen as a blue discoloration of the skin at the site of the intradermal injection (1) that progresses to necrosis. Histologically, the reaction is characterized by necrosis, interstitial hemorrhage, and thrombosis (2).

The hemorrhagic necrosis of the skin seen in meningococcal septicemia is histologically indistinguishable from the dermal Shwartzman reaction (3), suggesting that it may be due to an LSR-type reaction (4). Clinical observations in children with meningococcal septicemia indicate that peripheral ischemia can potentiate this type of hemorrhagic necrosis and suggest that a relatively brief period of ischemia, insufficient in itself to cause necrosis, may act in a similar manner to the

Received November 30, 2005; accepted March 14, 2006.

Correspondence: Anthony J. Penington, M.B.B.S., The Bernard O'Brien Institute of Microsurgery, 45 Fitzroy St, Fitzroy, VIC 3065, Australia; e-mail: a.penington@unimelb. edu.au

Financial support was provided by the Royal Australian College of Surgeons Foundation.

DOI: 10.1203/01.pdr.0000228326.99650.19 second provocative dose of endotoxin given intravenously in the LSR.

This article describes an attempt to test the hypothesis that ischemia can act as the provocative agent to produce the LSR. This is accomplished by an intradermal injection of endotoxin into the skin of a pedicled skin flap in the rat followed by a period of ischemia insufficient by itself to cause necrosis in an attempt to model the skin loss associated with meningococcemia.

\section{MATERIALS AND METHODS}

All experiments were conducted with the prior approval of the St Vincent's Hospital Animal Ethics Committee. Sprague-Dawley rats weighing between 200 and $300 \mathrm{~g}$ were used. Animals were anesthetized with either inhalational halothane ( $2 \%$ with oxygen) for injections or intraperitoneal pentobarbitone $(30 \mathrm{mg} / \mathrm{kg}$ ) for surgery. Animals were caged individually under standard environmental conditions and allowed food and water ad libitum.

Surgical technique. Inferior epigastric flaps of abdominal skin measuring $3 \times 3 \mathrm{~cm}^{2}$ were elevated bilaterally as previously described (5). If the flap is to be rendered ischemic, a microvascular clamp is applied to the pedicle and occlusion confirmed by microscopic examination. The flap is then sutured back in its original site. After $6 \mathrm{~h}$, a portion of the wound is opened and the clamp withdrawn. Return of flow is confirmed, the wound resutured, and the animal awoken.

Preliminary study. To determine the baseline sensitivity of rat skin to ischemia, flaps were elevated in 10 animals. Ischemia was induced for $6 \mathrm{~h}$ $(n=2), 8 \mathrm{~h}(n=2), 10 \mathrm{~h}(n=2)$, or $14 \mathrm{~h}(n=4)$ by clamping the vascular pedicle. The flaps were examined $48 \mathrm{~h}$ later.

Stage 1. In eight rats, bilateral inferior epigastric flaps were outlined. Within each flap three injection sites were marked, $1 \mathrm{~cm}$ apart and $1 \mathrm{~cm}$ from the nearest edge of the flap. An intradermal injection of $0.1 \mathrm{~mL}$ of endotoxin (Escherichia coli serotype 0111:4B) (Sigma Chemical Co., St. Louis, MO) at $0.1 \mathrm{mg} / \mathrm{mL}$ in normal saline was made into site 1 in each of the flaps.

Stage 2. Twenty-four hours later, an injection of endotoxin or normal saline was made in sites 2 and 3 , respectively, within each flap immediately before the flaps were raised. A microvascular clamp was applied to the pedicle of the experimental flap and visually inspected to confirm occlusion. The flap was then resutured into position. The control flap was raised and resutured without application of a clamp.

Stage 3. Six hours later, a 1-cm segment of suture was removed. The clamp was released from the pedicle of the experimental flap and inspected to confirm reflow.

Stage 4. Animals were killed either $30 \min (n=4)$ or $48 \mathrm{~h}(n=4)$ later and full-thickness tissue encompassing each injection site removed and fixed in $10 \%$ formol saline for histologic examination.

Abbreviations: LSR, local Shwartzman reaction 
Two additional animals underwent the sequence after 24-h preischemia injections were made into all three sites of each flap. After $6 \mathrm{~h}$ of ischemia, injection sites were biopsied segmentally 5, 10, and $20 \mathrm{~min}$ after removal of the clamp.

Histology. Following fixation, specimens were processed into paraffin wax. Ten-micrometer sections were cut and stained with hematoxylin and eosin.

Electron Microscopy. Representative 1-mm core biopsies were taken from all three sites. Specimens were fixed by immersion in 1\% Karnovsky's solution, embedded in araldite, and sections examined by transmission electron microscopy in a Phillips EM 300 electron microscope (Royal Philips Electronics, The Netherlands).

\section{RESULTS}

Baseline sensitivity to ischemia. Forty-eight hours after induction of ischemia, 6-h flaps showed no abnormality, 8-h flaps appeared swollen, and 10-h flaps were grossly swollen and discolored but not necrotic. These changes were confirmed microscopically (data not shown). Thus, $6 \mathrm{~h}$ of ischemia was chosen for subsequent experiments.

Macroscopic findings. In four of eight rats, the site of 24-h preischemia injection of endotoxin showed a faint area of blue discoloration within $10 \mathrm{~min}$ of reperfusion. In three of four rats allowed to progress to 48 -h reperfusion, a $5-\mathrm{mm}^{2}$ area of necrosis was visible at this site (Fig. 1a).

No abnormality was apparent at the sites where endotoxin or saline were injected immediately before surgery after either $30 \mathrm{~min}$ or $48 \mathrm{~h}$ of reperfusion.

Microscopic findings. After $30 \mathrm{~min}$ of reperfusion, 24-h preischemia injections of endotoxin showed extensive hemor- rhage into all layers of the skin. Leukocyte emigration was visible (Fig. 1b). Hemorrhage was visible as early as $5 \mathrm{~min}$ after release of the clamps.

No hemorrhage was present at sites of nondelayed injection of endotoxin (Fig. 1c), saline (Fig. 1d), or any of the three sites in the control flap.

After $48 \mathrm{~h}$ of reperfusion, extensive hemorrhage was again present at all sites of the 24 -h preischemia endotoxin injection but not in the other sites. Disruption of endothelial lining was visible. Polymorphs were present in large numbers at sites of both delayed and nondelayed injection of endotoxin and in smaller numbers at sites of saline injection. In some sites of 24-h preischemia endotoxin injection, the epidermis was necrotic.

Electron microscopy in the 24-h preischemia group showed dense packing of the microvasculature and vacuolation in the endothelium (Fig. 1e). These changes were not seen in the other sites (Fig. 1f).

\section{DISCUSSION}

The findings of our study show that a period of ischemia, insufficient in itself to cause necrosis, causes a hemorrhagic necrotic response with the histologic features of the LSR at a site in rat skin where endotoxin was injected $24 \mathrm{~h}$ earlier. When either the endotoxin or saline were injected immediately before ischemia, no microscopic evidence of necrosis

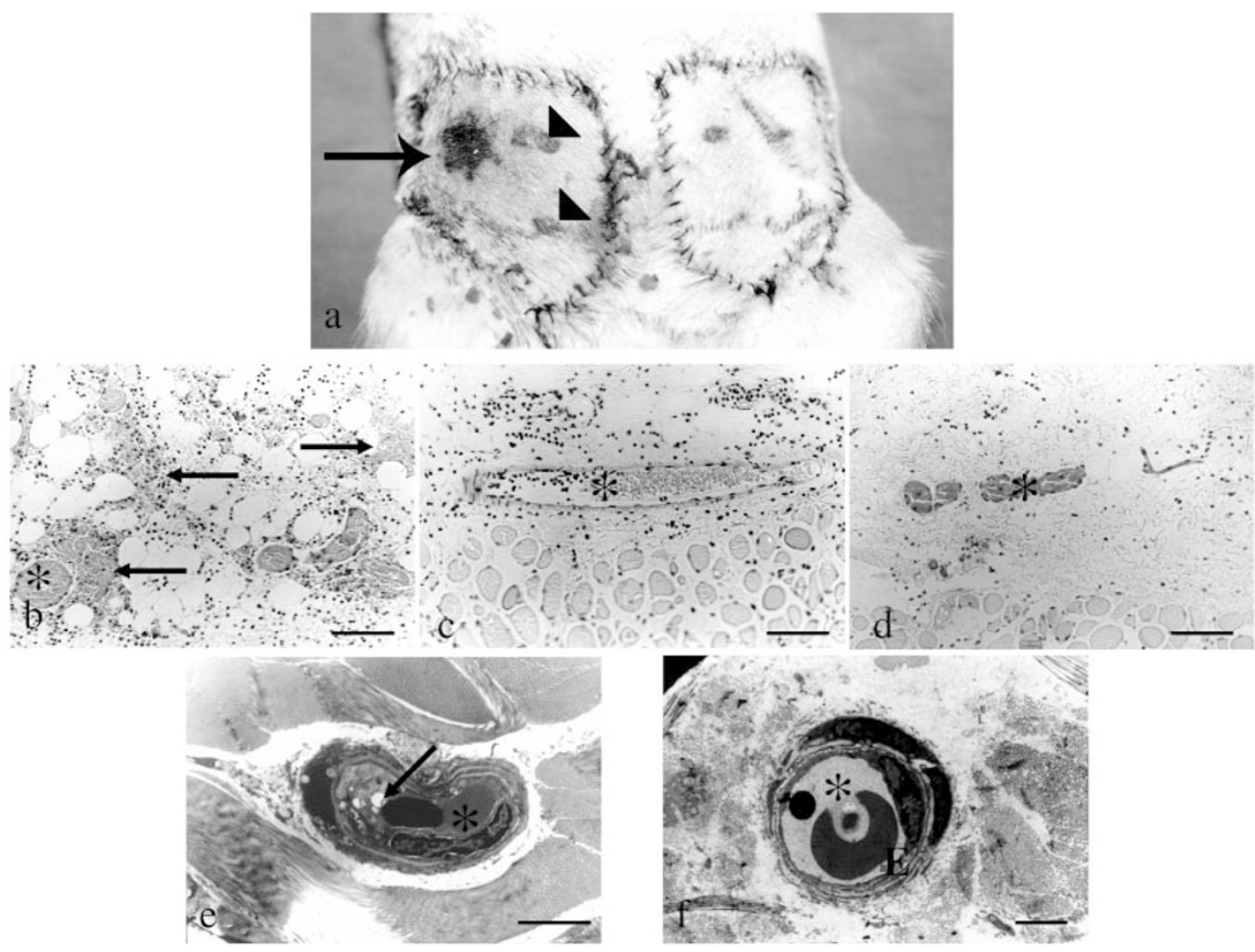

Figure 1. (a) Rat abdomen $48 \mathrm{~h}$ post ischemia demonstrating necrosis (arrow) at the site of endotoxin injection $24 \mathrm{~h}$ before ischemia. The other two injection sites show no evidence of necrosis (arrowheads). $(b-d)$ Hematoxylin and eosin stained 5- $\mu$ m-thick paraffin sections of rat skin. (b) A rapid and extensive hemorrhage (arrows) is present in the skin. Asterisk, blood vessel; scale bar $=100 \mu \mathrm{m}$. ( $c$ and $d$ ) Little or no hemorrhage present in the site of $(c)$ the endotoxin injection or $(d)$ saline injection. Asterisks, blood vessels; scale bars $=100 \mu \mathrm{m}$. ( $e$ and $f$ ) Transmission electron microscopy. (e) Occlusion of a capillary (asterisk) and vacuolation of endothelial cells (arrow); scale bar $=1 \mu \mathrm{m}$. $(f)$ Patent capillary lumen (asterisk) and normal endothelium $(E)$ in rat skin lesion where the endotoxin injection was immediately before ischemia; scale bar $=1 \mu \mathrm{m}$. 
was evident. This demonstrates that bacterial infection can sensitize tissues to the effects of ischemia, effectively lowering the threshold at which irreversible cell injury occurs. This interaction may relate to the pathogenesis of skin necrosis associated with meningococcemia.

Dermal lesions are seen in more than $50 \%$ of cases of meningococcemia (8). Although the pathogenesis is not fully understood, their similarity to the LSR has led some to postulate that the two processes share a common mechanism. The skin sites are thought to be prepared in the bacteremic phase of the disease (6), with the meningococci initiating a local vascular reaction with accumulation of neutrophils. Granulocytes enhance the inflammatory process, increasing the small vessel damage (6). The importance of neutrophil invasion in the LSR has been demonstrated by studies confirming that it only occurs if adequate numbers of polymorphs are present (7).

The LSR requires a second stimulus $24 \mathrm{~h}$ after the preparatory inoculation, consistent with the findings of this study. Previous theories have held that this provocative dose may be supplied by circulating endotoxin from persistent bacteremia associated with meningococcemia (8). However, our model demonstrates that local skin necrosis can be achieved with ischemia as the provocative agent $24 \mathrm{~h}$ after endotoxin exposure. The inoculating dose of endotoxin given at a defined period before the provocative insult may indicate upregulation of the inflammatory cascade, lowering the threshold for further expression of inflammatory mediators and subsequent necrosis from ischemia. However, it is possible that injection of endotoxin itself recruits a sufficient number of neutrophils locally to cause coagulation and tissue damage in the face of ischemia and reperfusion. Further work is needed to elucidate the role and source of granulocytes in this model, as well as further experiments with cytokine inhibition to clarify the involvement of mediators of ischemia-reperfusion injury.

To our knowledge, our study is the first to demonstrate that ischemia can act as the provocative agent to successfully produce the LSR, creating a novel model for the study the skin lesions associated with meningococcemia.

Acknowledgments. The authors thank the staff of the EMSU at St. Vincent's Hospital for their assistance, Rosalind Romeo for assistance with histology, and Andrew Morritt for his help with photography.

\section{REFERENCES}

1. Shwartzman G 1928 A new phenomenon of local skin reactivity to B. typhosus culture filtrate. Proc Soc Exp Biol Med 25:560-561

2. Ramirez-Romero R, Brogden KA 2000 The potential role of the Arthus and Shwartzman reactions in the pathogenesis of pneumonic pasteurellosis. Inflamm Res 49:98101

3. Davis CE, Arnold K 1974 Role of meningococcal endotoxin in meningococcal purpura. J Exp Med 140:159-171

4. Francis RB Jr 1990 Acquired purpura fulminans. Semin Thromb Hemost 16:310-325

5. Willemart G, Knight KR, Morrison WA 1998 Dexamethasone treatment prior to reperfusion improves the survival of skin flaps subjected to secondary venous ischaemia. Br J Plast Surg 51:624-628

6. Dahle JS 1983 Pathogenesis of hemorrhagic skin lesions in meningococcal disease. NIPH Ann 6:49-53

7. Argenbright LW, Barton RW 1992 Interactions of leukocyte integrins with intercellular adhesion molecule 1 in the production of inflammatory vascular injury in vivo. The Shwartzman reaction revisited. J Clin Invest 89:259-272

8. Hjort PF, Rapaport SI 1965 The Shwartzman reaction: pathogenetic mechanisms and clinical manifestations. Annu Rev Med 16:135-168 